\title{
Revista de Historia de la Psicología
}

www.revistahistoriapsicologia.es

\section{Análisis de la producción y colaboración interinstitucional en la psicología española desde una perspectiva de género entre 1970 y 1989.}

\author{
Francisco González Sala, Julia Osca-Lluch y Carmel Ferragut Domingo \\ Universidad de Valencia, Valencia, España
}

I N F O R M A C I Ó N A R T.

Recibido: 5 noviembre 2021

Aceptado: 23 febrero 2022

Palabras clave

Colaboración científica,

Género,

Psicología,

Historia de la ciencia,

Bibliometría

\begin{abstract}
R E S U M E N
El objetivo del presente estudio fue conocer las relaciones de colaboración entre instituciones y entre profesionales, así como su producción científica en función del género entre los años 1970 y 1989 en la Psicología española. Se analizaron 2.891 artículos, encontrándose una mayor colaboración en psicología entre investigadores de una misma institución y entre profesionales de la misma área. Desde una perspectiva de género, si bien los hombres tienen una mayor presencia y firman un mayor número de trabajos, se observa un aumento progresivo de las mujeres en la investigación psicológica, el cual se concreta en un mayor número de artículos firmados por hombres y mujeres y de trabajos firmados solo por mujeres. A modo de conclusión cabe mencionar la relevancia de incluir estudios de género desde una concepción social de la ciencia, permitiendo de este modo visibilizar las contribuciones de las mujeres en un área del conocimiento determinada.
\end{abstract}

\section{Analysis of the production and inter-institutional collaboration in Spanish psychology from a gender perspective between 1970 and 1989.}

\section{A B S T R A C T}

The object of the present study is to know the collaboration relationships between institutions and among professionals, as well as their scientific production based on gender between the years 1970 and 1989 in Spanish Psychology. There were 2.891 articles found, having a greater collaboration in Psychology between the researchers of a same institution and area of knowledge. From a gender perspective, even if male have a greater presence and sign a greater amount of papers, a progressive increase done by female researchers is observed. Particularly there is a greater number of articles published by men and women than works published exclusively by women. As a conclusion, it is worth mentioning the relevance of including gender perspective studies from a social conception within science, allowing by doing so to highlight women's contributions in a determined area of knowledge.
Scientific collaboration; Gender;

Psychology; History of Science;

Bibliometrics

\begin{abstract}
Francisco González Sala (10 https://orcid.org/0000-0003-4124-7459 Dpto. Psicología Evolutiva y de la Educación. Universidad de Valencia. Avda. Blasco Ibáñez, 21, 46019, Valencia (España). FAX: 963864671. Telf. 963983881. E-mail: Francisco.Gonzalez-Sala@uv.es

Julia Osca-Lluch (i) https://orcid.org/0000-0002-0449-5878 INGENIO (CSIC-Universitat Politècnica de València). Camino de Vera, s/n, 46022 València, (España). E-mail: juosllu@ingenio.upv.es

Carmel Ferragut Domingo (i) https://orcid.org/0000-0002-9756-9257 Departamento de Historia de la Ciencia y Documentación. Instituto de Historia de la Medicina y de la Ciencia López Piñero, Universidad de Valencia-CSIC. E-mail: Carmel.Ferragud@uv.es
\end{abstract}




\section{Introducción}

Conocer el desarrollo de una disciplina científica a lo largo de los años supone analizar, entre otras variables, diferentes indicadores bibliométricos, tanto de naturaleza cuantitativa, como por ejemplo el número de trabajos publicados por investigadores pertenecientes a una misma área del conocimiento y de un país o región determinada (González-Sala y Osca-Lluch, 2018), el número de trabajos en colaboración (González-Sala et al., 2021; López Ferrer y Osca-Lluch, 2009) o el número de hombres y mujeres firmantes de trabajos (Barrios et al., 2013; González-Sala y Osca-Lluch, 2018), como de naturaleza cualitativa, al considerar, como ejemplo, las relaciones entre instituciones o profesionales de diferentes áreas que firman trabajos en colaboración.

A finales del siglo XIX y principios del siglo XX cobra fuerza la psicología científica con la creación en el año 1879 del primer instituto de Psicología Experimental en la Universidad de Leipzig. En el caso concreto de España este hecho se produce con la celebración del Primer Congreso de la Asociación Española para el Progreso de la Ciencias celebrado en 1908 (Ibarz, 2013). Tras la Guerra Civil española se produce un cambio hacia una psicología más filosófica, o como apunta Carpintero (1984), una psicología escolástica y tomista, dirigida principalmente por la figura de un dominico, el padre Barbado, que ocuparía la cátedra de Psicología Experimental. Todo el proceso estuvo marcado por el catolicismo imperante (López, 2016). En este sentido, como apunta Germain (1980) el impacto de la Guerra civil supuso un freno en el desarrollo de la psicología en España.

Pérez-Delgado et al. (1981) señalan la influencia procedente de la Medicina y de la Fisiología, principalmente, en el desarrollo de la Psicología hacia las Ciencias Experimentales. Estos autores diferencian dos periodos en los que se puede enmarcar las aportaciones de la Medicina y la Psiquiatría a la Psicología. El primero comprende desde mitad del siglo XVIII hasta la mitad del siglo XIX. Este periodo se caracteriza por las aportaciones de Franz Gall con la frenología y las aportaciones de psiquiatras como Pinel, Rush, Esquirol, Itard o Seguin, que atribuyeron diferentes enfermedades mentales a causas neurobiológicas y, principalmente, por las figuras de Messmer, Elliotson, Brais o Esdaile, que introdujeron la hipnosis como técnica en la Medicina. En el segundo periodo destacan figuras como Ramón y Cajal, Golgi, Fritsch, Hitzig o Jackson al abordar el estudio del sistema nervioso central, y la de los psiquiatras Princce, Kraepelin, Bleuler o Wiersma. Esta relación de la Psicología con la Psiquiatría tras la Guerra Civil es señalada por Carpintero (2006) en su monografía sobre la historia de la psicología en España.

Los estudios de Psicología comienzan a impartirse en la Facultad de Filosofía y Letras en la Universidad Complutense de Madrid en el año 1968 (Tortosa-Pérez et al. 2021), por lo que no será hasta tres años después cuando salga la primera promoción. Casi una década más tarde, en 1980, se crea el Colegio Oficial de Psicólogos (Ley 43/1979, de 31 de diciembre, BOE el 8 de enero de 1980) y las Facultades de Psicología (Real Decreto 1652/1979, de 25 de mayo, BOE de 5 de julio). Estos dos acontecimientos invitan a pensar en un cambio en la Psicología como ciencia y como profesión en España, pudiendo diferenciar un periodo antes del reconocimiento oficial de la profesión que comprendería entre 1970 y 1979 y un periodo posterior, a partir del 1980.

Según García (2005) la década de los años 70 se caracteriza por el crecimiento, difusión, diversidad, pluralidad, profesionalización e institucionalización de la Psicología, con el objetivo de dotar a la Psicología de un estatus científico propio (Carpintero y Tortosa, 1996). Para Vera (2005) es 1979 el año clave en el devenir de la Psicología española por el posicionamiento social e institucional de ésta. TortosaPérez et al. (2021) apuntan ese mismo año como clave en la psicología española a raíz de la creación del Colegio Oficial de Psicólogos. En esta misma línea, en el VI Congreso Nacional de Psicología y de la Asamblea General del S.E.P. se recomienda una formación del profesorado caracterizada por el rigor científico, una investigación de calidad y la necesidad de una formación científico-académica (Caparrós, 1990), lo que contribuirá a una mayor presencia de la Universidad en la producción científica, así como al nacimiento de un gran número de revistas científicas de psicología (Alcaín y Ruíz-Gálvez, 1998), muchas de las cuales son editadas por departamentos universitarios.

El periodo entre 1970 y 1989, se caracteriza por un aumento de la producción y de la colaboración científica en psicología, principalmente en los años finales, llegando incluso a superar el número de trabajos en colaboración al de artículos de autoría única (González-Sala et al., 2021). Esta tendencia se mantiene entre los años 1992 y 2001, siendo el porcentaje de trabajos firmados en colaboración por autores españoles del 72.79\% (López Ferrer y Osca-Lluch, 2009).

La colaboración científica, entendida como el trabajo conjunto entre profesionales, puede estudiarse desde una perspectiva social de la ciencia, la cual entiende la ciencia como una actividad social, siendo el conocimiento, un bien social, donde los descubrimientos y avances, pero también los cambios de paradigmas, son parte de la historia de la humanidad. Como actividad social, cabe incluir también las relaciones entre científicos y científicas, formando equipos de investigación inter e intra disciplinarios, los cuales a su vez tienen también una repercusión en el avance del conocimiento y en el devenir de las disciplinas científicas, apareciendo nuevas áreas. En este sentido algunos trabajos han estudiado esta colaboración desde la interdisciplinaridad (Morillo et al., 2003; Rafols y Meyer, 2007; Rafols y Meyer, 2010), siendo ésta una de las variables de estudio en el presente trabajo. Para González Alcaide y Gómez Ferri (2014) el análisis de la colaboración científica en todas sus dimensiones, sociales, organizacionales, económicas, entre otras, es una forma de entender la ciencia en general. Atendiendo a la hipótesis de Carpintero (1980) que considera la ciencia como una organización, se entiende la relevancia de la colaboración científica dentro de esta organización, entendiendo esta colaboración desde la horizontalidad frente a la verticalidad. Esta organización social de la ciencia asociada a la colaboración científica ya fue señala por Robert Merton (Merton, 1977).

Carnes y Griffen (1990) destacan la relevancia del género como herramienta en el estudio de la historia de la ciencia. Desde una perspectiva de género, la mujer ha contribuido desde los primeros momentos al desarrollo de la Psicología (Wilson, 2003), si bien éstas han permanecido en el anonimato (Scarborough y Furumoto, 1987), enfrentándose a toda una serie de condicionantes sociales y académicos que han dificultado el desarrollo de su profesión (Furumoto, 1992; García Dauder, 2005). 
En el caso de la Psicología española, Barrios et al. (2013) encuentran que las mujeres firman un menor número de trabajos como primera firmante; aproximadamente en el $42 \%$ de los artículos publicados la primera firmante era una mujer. González-Sala y Osca-Lluch (2018) señalan una igualdad de género entre hombres y mujeres con respecto a la producción científica y como primer firmante, si bien los hombres firman más trabajos en revistas situadas entre los primeros cuartiles. Otros trabajos señalan una baja productividad de las mujeres en la psicología española (Olivas-Ávila y Musi-Lechuga, 2010) o como apunta González-Alcaide et al. (2010) en el caso de los trabajos publicados en la revista Psicothema entre 1989 y 2008. Por su parte, en el campo de la psicología educativa en España entre 2008 y 2018, González-Sala, Haba-Osca et al. (2021) encuentran un mayor número de firmas procedentes de mujeres, si bien existe un mayor número de hombres como grandes productores. Otros estudios relacionados con la ciencia en general en España señalan como las mujeres publican menos trabajos y estos reciben un menor número de citas, firmando en menor medida como primer firmante (FECYT, 2017).

Esta mayor presencia de hombres como grandes productores ha mantenido en el anonimato las aportaciones de las mujeres en materia de producción científica, en tanto que los estudios realizados no han tenido en consideración una perspectiva de género diferenciando entre hombres y mujeres, sirviéndose de clasificaciones generalistas donde, al parecer, lo único relevante era el número de trabajos firmados. En este sentido cabe considerar que las mujeres presentan una menor frecuencia de visitas a centros fuera del país de procedencia; son visitas más cortas y más próximas al lugar de residencia, dándose principalmente al principio de sus carreras investigadoras $\mathrm{y} / \mathrm{o}$ docente (Cañibano et al., 2016), lo que deja entrever el papel que puede desempeñar el ser madre y la crianza en una menor producción científica si se tiene en cuenta, tal y como apuntan De Filippo et al. (2009) que son las mujeres sin hijos las que presentan una mayor movilidad internacional, lo que a su vez puede condicionar el número y tipo de colaboraciones entre profesionales. En este sentido, Araujo y Fontainha (2017) señalan como en los trabajos firmados solo por mujeres el número de firmantes suele ser también menor.

El primer objetivo del presente estudio fue analizar la colaboración inter e intra institucional y la colaboración inter e intra profesional en la Psicología española a través de las instituciones de procedencia de los firmantes de artículos y revisiones entre 1970 y 1989. Como segundo objetivo, partiendo desde una perspectiva de género, se procedió a analizar la producción científica en este periodo según diferentes indicadores bibliométricos y a visualizar a los y las principales productores y productoras en función del género.

\section{Método}

\section{Materiales y Procedimiento}

Para el presente estudio se seleccionaron los trabajos cuya tipología documental fueran artículos científicos, de revisión o de discusión. Además, debían estar firmados al menos por un autor perteneciente a una institución española de psicología o que tuvieran formación psicológica, descartándose aquellos trabajos que únicamente estaban firmados por autores extranjeros o por autores no psicólogos.

Tabla 1. Número de artículos por revista.

\begin{tabular}{|c|c|}
\hline Revistas & № artículos \\
\hline $\begin{array}{l}\text { Actas Luso-Españolas de Neurología, Psiquiatría y Ciencias } \\
\text { Afines }\end{array}$ & 19 \\
\hline Análisis y Modificación de Conducta & 265 \\
\hline Anales de Psicología & 42 \\
\hline Anuario de Psicología & 194 \\
\hline Apuntes de Psicología & 69 \\
\hline Boletín de Psicología & 94 \\
\hline Clínica y Análisis Grupal & 94 \\
\hline Cuadernos de Psicología & 106 \\
\hline Estudios de Psicología & 190 \\
\hline Evaluación Psicológica/Assessment Psychology & 45 \\
\hline Infancia y Aprendizaje & 197 \\
\hline Informació Psicològica & 131 \\
\hline Informes de Psicología & 110 \\
\hline Papeles del Psicólogo & 114 \\
\hline Psicológica & 162 \\
\hline Psicologemas & 34 \\
\hline Psicothema & 7 \\
\hline Revista Española de Psicoterapia Analítica & 3 \\
\hline Revista Española de Terapia del Comportamiento & 80 \\
\hline Revista de Investigación Psicológica & 28 \\
\hline Revista de Psicología de la Educación & 12 \\
\hline Revista de Historia de la Psicología & 168 \\
\hline Revista de Psicología General y Aplicada & 521 \\
\hline Revista de Psicología del Trabajo y de las Organizaciones & 42 \\
\hline Revista de Psicología Universitas Tarraconensis & 102 \\
\hline Revista de Psicoterapia y Psicología Social Aplicada & 5 \\
\hline Revista de Psicoterapia y Psicosomática Médica & 12 \\
\hline Revista de Psicología de la Salud & 12 \\
\hline Revista de Psicología Social & 35 \\
\hline Total de trabajos & 2891 \\
\hline
\end{tabular}


El número de trabajos recuperados para este estudio fue de 2.891, los cuales fueron publicados entre 1970 y 1989 en 29 revistas diferentes. En la tabla 1 se recoge el número de artículos por revista. En concreto, en el periodo entre 1970 y 1974 se seleccionaron 92 artículos, en el periodo entre 1975 y 1979 un total de 323 artículos, entre 1980 y 1984 fueron 939 trabajos y entre 1985 y 1989 el número de trabajos fue de 1535.

La selección de los años objeto de estudio se realizó teniendo en cuenta dos hechos históricos en la psicología española, tal y como se ha comentado en párrafos anteriores. Por un lado, la primera promoción de psicólogos y psicólogas, a principios de los años 70 y la aprobación de los Colegios Oficiales de Psicología y las Facultades de Psicología en 1980. Según estas fechas, se decidió analizar un periodo de 20 años considerando los 10 años antes y después de 1980, año en el que se puede hablar del reconocimiento oficial de la Psicología como profesión.

Con respecto al procedimiento seguido, se seleccionaron en primer lugar las revistas objeto de estudio. Esta selección se realizó siguiendo dos criterios, por un lado se consultaron artículos científicos que hubieran abordado el desarrollo de la psicología española a través de las revistas científicas como el trabajo de Tortosa y Civera (2001) o el de Alcaín y Ruíz-Galvez (1998) y, por otro lado, se consultaron los títulos de las revistas de psicología española que recogía el Colegio Oficial de Psicólogos, la Web of Science y Scopus para posteriormente conocer si ya eran editadas entre 1970 y 1989. Posteriormente, se procedió a consultar de forma electrónica o física los trabajos seleccionados en las revistas incluidas en el estudio en las bibliotecas de las facultades de Psicología y de Medicina de la Universitat de València.

De cada uno de los trabajos consultados se recogió en una base de datos diseñada ad hoc el nombre y apellidos del autor o autora, la filiación y el género de los autores. Posteriormente, se normalizaron los nombres de los autores así como la afiliación institucional.

Por último, se procedió a la obtención de los resultados tras definir las variables objeto de estudio y a su posterior interpretación.

\section{Variables}

Se analizaron el número de artículos según el tipo de colaboración, diferenciando entre colaboración institucional la cual incluía dos categorías. Por un lado, Colaboración intrainstitucional cuando todos los firmantes pertenecen a una misma institución y, por otro, Colaboración interinstitucional cuando los firmantes provienen al menos de dos instituciones diferentes. Además, se diferenció entre Colaboración intraprofesional, cuando todos los firmantes eran psicólogos o psicólogas, y Colaboración interprofesional, cuando el artículo era firmado por un psicólogo o psicóloga y por otro autor perteneciente a otra área del conocimiento.

Con relación al género se tuvo en consideración el número de autores hombres y mujeres, así como el número de firmas y la composición de miembros firmantes de un mismo trabajo, diferenciando entre artículos firmados solo por hombres, artículos firmados solo por mujeres y artículos firmados por hombres y mujeres.

Los casos en los que no se pudo determinar el género de los autores o la institución de procedencia se categorizaron como desconocidos, recogiéndose en número de casos y el porcentaje, si bien no fueron incluidos en el estudio de diferencias estadísticas entre las variables de comparación.

La variable años se categorizó en cuatro periodos temporales de cinco años (1970-1974, 1975-1979, 1980-1984 y 1985-1989). Por último, se recopilaron los nombres de los mayores productores en cada periodo estudiado, diferenciando entre hombres y mujeres.

\section{Análisis}

Se realizaron recuentos de frecuencias y cálculo de porcentajes para las variables objeto de estudio. A la hora de establecer diferencias estadísticas para variables categóricas se realizaron pruebas $\chi 2$. Todo ello se llevó a cabo mediante el paquete estadístico SPSS 25.

\section{Resultados}

\section{Análisis de la colaboración interinstitucional en la psicología española entre 1970 y 1989.}

Tal y como puede observarse en la Tabla 2, en todos los periodos analizados existe un mayor porcentaje de artículos firmados en colaboración entre miembros de una misma institución, porcentaje que se sitúa en un 73.8\%, siendo el periodo entre 1975 y 1979 donde el porcentaje de colaboración intrainstitucional es menor con un 59.2\%. Estas diferencias resultaron ser estadísticamente significativas $\chi^{2}=$ 16.615, 3 gl., $\mathrm{p}=.001, \Phi=.119$.

Con respecto a la colaboración intraprofesional, alrededor del $85 \%$ de las colaboraciones son entre psicólogos a lo largo del periodo estudiado. Estas diferencias resultaron ser estadísticamente significativas $\chi 2=85.531,3$ gl., $\mathrm{p}=.000, \Phi=.276$.

Es en el periodo entre 1975 y 1979 cuando existe una mayor colaboración entre psicólogos y psicólogas con profesionales de otras disciplinas, siendo el porcentaje de colaboración interprofesional del 29.6\%. Esta colaboración principalmente es con psiquiatras (19 trabajos), con médicos (9 trabajos) y un trabajo firmado con una maestra.

En el periodo entre 1980 y 1984, de los 23 trabajos firmado en colaboración entre diferentes profesionales 15 son firmados por psicólogos juntamente con psiquiatras o médicos, 5 trabajos con profesionales de la pedagogía, un trabajo con una maestra y dos trabajos con otros profesionales.

En el periodo entre 1985 y 1989 los 36 trabajos en colaboración interprofesional son firmados principalmente por psicólogos en colaboración con psiquiatras (14 trabajos), con médicos (6 trabajos), con pedagogos o pedagogas (5 trabajos) y 11 trabajos con otros profesionales con titulaciones en Farmacia, Trabajo Social, Biología, Enfermería o Filosofía.

Por último, en el periodo entre 1970 y 1974 solo dos trabajos cuentan con colaboración interprofesional, si bien cabe destacar que los autores son médicos y cuentan con formación en Psicología como son las figuras de José Germain y José Corominas Busqueta. Esta 
Tabla 2. Colaboración institucional y profesional en la Psicología española entre 1970 y 1989.

\begin{tabular}{lccccccc} 
Años & $\begin{array}{c}\text { Artículos } \\
\text { colaboración }\end{array}$ & $\begin{array}{c}\text { Col. intra } \\
\text { institucional }\end{array}$ & $\begin{array}{c}\text { Col. inter } \\
\text { institucional }\end{array}$ & D & $\begin{array}{c}\text { Col. intra } \\
\text { profesional }\end{array}$ & $\begin{array}{c}\text { Col. inter } \\
\text { profesional }\end{array}$ & D \\
\hline $1970-1974$ & 20 & $14(70.0 \%)$ & $2(10.0 \%)$ & $4(20.0 \%)$ & $17(85.0 \%)$ & $2(10.0 \%)$ & $1(5.0 \%)$ \\
\hline $1975-1979$ & 98 & $58(59.2 \%)$ & $32(32.6 \%)$ & $8(8.2 \%)$ & $58(59.2 \%)$ & $29(29.6 \%)$ & $11(11.2 \%)$ \\
\hline $1980-1984$ & 321 & $225(70.1 \%)$ & $72(22.4 \%)$ & $24(7.8 \%)$ & $272(84.7 \%)$ & $23(7.2 \%)$ & $26(8.1 \%)$ \\
\hline $1985-1989$ & 785 & $605(77.1 \%)$ & $145(18.5 \%)$ & $35(4.4 \%)$ & $700(89.3 \%)$ & $37(4.7 \%)$ & $48(6.0 \%)$ \\
\hline Total & $\mathbf{1 2 2 4}$ & $\mathbf{9 0 4}(\mathbf{7 3 . 8 \% )}$ & $\mathbf{2 5 1 ( 2 1 . 0 \% )}$ & $\mathbf{6 9 ( 5 . 2 \% )}$ & $\mathbf{1 0 4 7}(\mathbf{8 5 . 6 \% )}$ & $\mathbf{9 1 ( 7 . 4 \% )}$ & $\mathbf{8 6}(\mathbf{7 . 0 \% )}$ \\
\hline
\end{tabular}

misma situación se repite con otras figuras como Antonio Guillamón Fernández, doctor en Medicina y catedrático de Psicobiología, o Jorge L. Tizón que es psiquiatra, psicólogo y neurólogo.

\section{Análisis de la producción científica desde una perspectiva de género.}

\section{Evolución del número de artículos y revisiones entre 1970 y 1989 según el género de los firmantes.}

Tal y como viene recogido en la tabla 3, el porcentaje de artículos firmados por hombres es mayor en todo el periodo analizado, con porcentajes entre el 68\% y el 52\% aproximadamente. En el caso de los artículos firmados sólo por mujeres los porcentajes comprenden entre un $25 \%$ y un $15 \%$ aproximadamente, disminuyendo el número de trabajos en el periodo comprendido entre 1975 y 1984. Por su parte, se observa con los años un crecimiento de los trabajos firmados por hombres y mujeres, pasando de un 5.4\% en el periodo entre 1970-1974 a un 25\% entre los años 1985 y 1989. Estas diferencias resultaron ser estadísticamente significativas $\chi 2=94.758,6$ gl., $\mathrm{p}=.000, \Phi=.183$.

Cuando se analiza el género de los firmantes de aquellos artículos con un solo autor, tres cuartas partes de los trabajos son firmados por hombres en el periodo comprendido entre 1970 y 1989. Esta mayor presencia de firmas de hombres se mantiene en los cuatro periodos temporales analizados, si bien con los años se observa un incremento del porcentaje de mujeres que firman como única autora, pasando del

Tabla 3. Número de artículos en función del género de los firmantes entre 1970 y 1989.

\begin{tabular}{|c|c|c|c|c|c|}
\hline Años & Total artículos & № y \% art. solo por hombres & № y \% de art. solo por mujeres & № y \% art. mixtos & D \\
\hline 1970-1974 & 92 & $60(65.2 \%)$ & $23(25.0 \%)$ & $5(5.4 \%)$ & $4(4.4 \%)$ \\
\hline 1975-1979 & 323 & $220(68.1 \%)$ & 48 (14.9\%) & $40(12.4 \%)$ & $15(4.6 \%)$ \\
\hline 1980-1984 & 939 & $640(68.1 \%)$ & $158(16.8 \%)$ & $128(13.6)$ & $13(1.5 \%)$ \\
\hline 1985-1989 & 1537 & $806(52.4 \%)$ & $303(19.7 \%)$ & $384(25.0 \%)$ & $44(2.9 \%)$ \\
\hline Total & 2891 & 1726 (59.7\%) & $532(18.4 \%)$ & $557(19.2 \%)$ & $76(2.7 \%)$ \\
\hline
\end{tabular}

Nota: D=número de artículos en los que no se pudo determinar el género de los firmantes.

Tabla 4. Número de artículos de autoría única en función del género del autor entre 1970 y 1989.

\begin{tabular}{|c|c|c|c|c|}
\hline Periodo & Total artículos autoría única & Art 1 solo firmante hombre & Art 1 solo firmante mujer & D \\
\hline $1970-1974$ & 72 & $54(75.0 \%)$ & $17(23.6 \%)$ & $1(1.4 \%)$ \\
\hline 1975-1979 & 225 & $180(80.0 \%)$ & $40(17.8 \%)$ & $5(2.2 \%)$ \\
\hline 1980-1984 & 618 & $486(78.6 \%)$ & $126(20.4 \%)$ & $6(1.0 \%)$ \\
\hline 1985-1989 & 752 & $538(71.5 \%)$ & $204(27.1 \%)$ & $10(1.4 \%)$ \\
\hline Total & 1667 & $1258(75.5 \%)$ & $387(23.2 \%)$ & $22(1.3 \%)$ \\
\hline
\end{tabular}


Tabla 5. Número y porcentaje de firmas de hombres y mujeres entre 1970 y 1989.

\begin{tabular}{|c|c|c|c|c|c|c|c|c|}
\hline Periodo & $\begin{array}{c}\text { Firmas } \\
\text { hombres }\end{array}$ & $\begin{array}{c}\text { Autores } \\
\text { hombres }\end{array}$ & $\begin{array}{l}\text { Firmas } \\
\text { mujeres }\end{array}$ & $\begin{array}{c}\text { Autores } \\
\text { Mujer }\end{array}$ & $\begin{array}{l}\text { Firmas } \\
\text { descon. }\end{array}$ & $\begin{array}{l}\text { Autores } \\
\text { descon. }\end{array}$ & $\begin{array}{c}\text { Total } \\
\text { firmas }\end{array}$ & $\begin{array}{c}\text { Total } \\
\text { autores }\end{array}$ \\
\hline 1970-1974 & 77 (64.2\%) & $53(57.6 \%)$ & 37 (30.8\%) & $30(32.6 \%)$ & $6(5.0 \%)$ & $9(9.8 \%)$ & 120 & 92 \\
\hline $1975-1979$ & $339(72.1 \%)$ & 190 (66.2\%) & $109(23.2 \%)$ & $77(26.8 \%)$ & $22(4.7 \%)$ & $20(7.0 \%)$ & 470 & 287 \\
\hline 1980-1984 & $981(71.0 \%)$ & $436(62.0 \%)$ & $360(26.0 \%)$ & $228(32.4 \%)$ & $41(3.0 \%)$ & $41(5.6 \%)$ & 1382 & 704 \\
\hline Total & 3188 (65.5\%) & 1454 (57.9\%) & 1474 (30.3\%) & 874 (34.8\%) & $202(4.2 \%)$ & $183(7.3 \%)$ & 4864 & 2510 \\
\hline
\end{tabular}

17.8\% entre 1975 a 1979 a un $27.4 \%$ entre los años 1985 y 1989. Estas diferencias resultaron ser estadísticamente significativas $\chi^{2}=12.682$, 3 gl., $\mathrm{p}=.005, \Phi=.088$. Todos estos datos pueden consultarse en la tabla 4.

\section{Análisis de las firmas en función del género entre 1970 y 1989.}

Cuando se analizan las firmas en función del género se puede observar una mayor presencia de firmas de hombres, un $65.5 \%$, frente a las firmas de mujeres (30.3\%) en todo el periodo estudiado, si bien cuando se analizan los datos en función de los diferentes periodos analizados se observa un crecimiento constante de firmas de mujeres, pasando de un $23.2 \%$ en el periodo $1975-1979$ a un $33.5 \%$ en el periodo 1985-1989. Todos estos datos pueden consultarse en la Tabla 5. Estas diferencias resultaron ser estadísticamente significativas $\chi^{2}=40.508$, $3 \mathrm{gl}, \mathrm{p}=.000, \Phi=.093$.

Con respecto al número de autores en función del género, existe un mayor número de hombres (57.9\%) que de mujeres (34.8\%), incrementándose el número de mujeres como firmantes de trabajos a lo largo de los años, pasando de un $26.8 \%$ a un $37.7 \%$ (ver tabla 5). Estas diferencias resultaron ser estadísticamente significativas $\chi^{2}=18.380$, 3 gl., $\mathrm{p}=.000, \Phi=.089$.

\section{Relación de autores y autoras con mayor producción según el periodo analizado}

Tal y como recoge la tabla 6 , se puede observar una mayor producción de trabajos en el caso de los hombres como grandes productores, si bien y teniendo en cuenta una perspectiva diferencial de género cabe destacar las mujeres que han contribuido al desarrollo de la Psicología española atendiendo a la producción científica.

En el caso de los hombres destacan figuras como Heliodoro Carpintero Capell de la Universidad de Valencia y Vicente Pelechano Barberá de la Universidad de la Laguna, al situarse como grandes productores en tres de los cuatro periodos temporales analizados. Con respecto a las mujeres, destacan las figuras de Rocío Fernández Ballesteros, de la Universidad Autónoma de Madrid, al situarse como una de las mayores productoras en tres de los periodos temporales analizados y Mạ José Báguena Puigcerver y Elena Ibáñez Guerra, ambas de la Universidad de Valencia, al estar consideradas como grandes productoras entre los años 1980 y 1989.

\section{Conclusiones}

A la hora de considerar tanto la colaboración científica como la producción científica desde una perspectiva de género cabe tener en cuenta toda una serie de factores históricos, como por ejemplo la creación de los colegios profesionales, aspectos políticos, en el caso concreto de la Psicología española vinculados a la transición que vivió el país en los últimos años de la década de los 70, o aspectos de índole social, como por ejemplo determinantes asociados a roles de género (García Dauder, 2005; Furumoto, 1992). Todos estos aspectos son considerados por Bucchi (2004) y Knorr-Cetina y Mulkay (1983) en los estudios sociales de la ciencia y por Carpintero (1980) y Merton (1977) al considerar la ciencia como una organización.

La colaboración científica es un imperativo en la ciencia actual, y en el caso concreto de la Psicología española una práctica común a partir del año 1987 donde los trabajos firmados en colaboración superan por primera vez a los trabajos firmados por un solo autor (GonzálezSala, Osca-Lluch et al., 2021), manteniéndose esta tendencia en años posteriores (López-Ferrer y Osca-Lluch, 2009). Si bien la colaboración científica aumenta con los años (González-Sala, Osca-Lluch et al., 2021), ésta se caracteriza por un aumento de la colaboración intrainstitucional e intraprofesional a partir del año 1980, siendo cada vez mayor en años posteriores, aspecto que puede explicarse por el reconocimiento de las Facultades de Psicología y de la creación del Colegio Oficial de Psicólogos en el año 1980, principalmente en relación a la colaboración intrainstitucional e intraprofesional. Por otro lado, el presente trabajo apunta mayores porcentajes de colaboración interinstitucional e interprofesional en el periodo entre 1975 y 1979 con porcentajes del $32.6 \%$ y del $29.6 \%$ respectivamente, lo cual puede ser explicado por la necesidad del reconocimiento de la profesión en nuestro país y por la apertura provocada por la transición política. Con respecto a la colaboración entre profesionales de diferentes áreas del conocimiento, es la Psiquiatría la que en mayor medida ha colaborado en la firma de trabajos con autores procedentes de la Psicología, tendencia que se mantiene a lo largo de los años analizados, colaboración que puede ser esperada si se tiene en cuenta el papel que jugaron figuras relevantes de la psiquiatría en el desarrollo de la psicología. 
Tabla 6. Autores y autoras con mayor producción científica según el periodo temporal analizado.

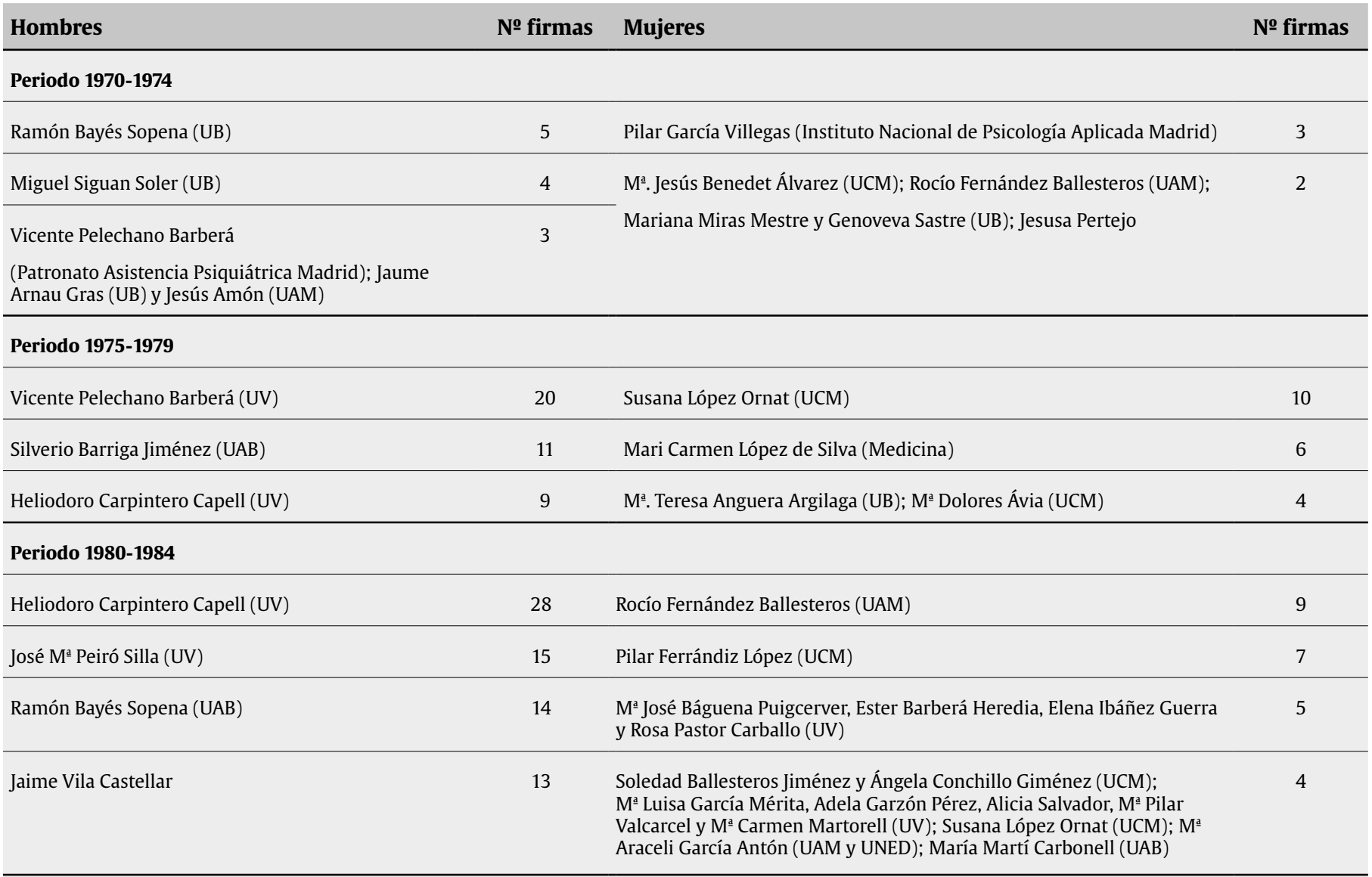

\begin{tabular}{|c|c|c|c|}
\hline Fco. Tortosa Gil (UV) & 30 & $\begin{array}{l}\text { Rocío Fernández Ballesteros y Carmen Vizcarro Guarch (UAM); Mª } \\
\text { Vicenta Mestre (UV) }\end{array}$ & 10 \\
\hline José Mª Peiró Silla (UV) & 25 & $\begin{array}{l}\text { Mª Victoria del Barrio, Adela Garzón Pérez, Elena Ibáñez Guerra y Mª. } \\
\text { Consuelo Roldán Badía (UV); Elena Quiñones Vidal (UM); Carmen } \\
\text { Vizcarro Guarch (UAM) }\end{array}$ & 9 \\
\hline Vicente Pelechano Barberá (UV y ULL) & 16 & Ma . Dolores Hierro Bustos (Facultad Tarragona) & 8 \\
\hline Fernando Silva Moreno (UCM) & 15 & $\begin{array}{l}\text { María Antonia Zalbidea, Edelia Villarroya Soler y Cristina Botella Arbona } \\
\text { (UV); María Dolores Avia (UCM) }\end{array}$ & 7 \\
\hline
\end{tabular}

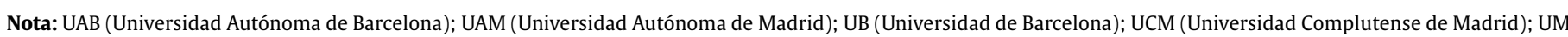
(Universidad de Murcia); UV (Universidad de Valencia); ULL (Universidad de la Laguna).

Desde una perspectiva de género, dada su importancia en los estudios de historia de la ciencia (Carnes y Griffen, 1990), cabe rescatar y visibilizar el papel de las mujeres en el desarrollo científico de la psicología en general (Wilson, 2003; Scarborough y Furumoto, 1987) y en la psicología española en particular, figuras que quedaban en segundo plano en estudios más generalistas sobre la producción científica. Entre estas pioneras destacan Rocío Fernández Ballesteros, de la Universidad Autónoma de Madrid, Susana López Ornat y María Dolores Ávia, de la Universidad Complutense de Madrid o María
José Báguena Puigcerver y Elena Ibáñez Guerra de la Universidad de Valencia, entre otras.

En este sentido, si bien existe un mayor número de autores hombres y un mayor número de firmas de hombres entre 1970 y 1989 , se observa un aumento con los años de las mujeres como autoras, representando el $37.7 \%$ del total en el último periodo, y en cuanto al número de firmas, con un 33.5\% de las firmas totales en el periodo entre 1985 y 1989. Esta mayor representación de las mujeres con respecto a la producción de artículos científicos se ha materializado 
principalmente en un aumento de los grupos mixtos, en los cuales participan tanto hombres como mujeres, y en el aumento de los trabajos firmados solo por mujeres, descendiendo el porcentaje de trabajos firmados únicamente por hombres. En este sentido, futuros trabajos han de abordar la evolución de la producción científica desde una perspectiva de género, confirmando o no la tendencia mostrada entre los años 1970 y 1989 en la psicología española.

Como principal limitación del presente estudio cabe mencionar el número limitado de revistas incluidas para el análisis de los trabajos teniendo en cuenta que no se han incluido otras revistas editadas fuera de España, en las cuales puede haber participación de autores españoles en este periodo analizado, si bien cabe destacar que el presente estudio es el que recoge un mayor número de revistas y documentos analizados en este periodo histórico de la psicología española, rescatando a partir de una perspectiva de género a las psicólogas que contribuyeron al desarrollo de la psicología en España a través de su producción científica.

\section{Referencias}

Alcaín, M. D. y Ruíz-Gálvez, M. (1998). Evolución de las revistas españolas de Psicología [Evolution of Spanish Psychology Journals]. Papeles del Psicólogo, 70 (junio), 35-42. http://www.papelesdelpsicologo.es/resumen?pii=785

Araujo, T. y Fontainha, E. (2017). The specific shapes of gender imbalance in scientific authorships: A network approach. Journal of Informetrics, 11, 88102. https://doi.org/10.1016/j.joi.2016.11.002

Barrios, M., Villarroya, A. y Borrego, A. (2013). Scientific production in psychology: a gender analysis. Scientometrics, 95(1), 15-23. https://doi. org/10.1007/s11192-012-0816-4.

Bucchi, M. (2004). Science in Society. Nueva York: Routledge.

Cañibano, C., Fox, M. F. y Otamendi, F. J. (2016). Gender and patterns of temporary mobility among researchers. Science and Public Policy, 43, 320-331. http:// dx.doi.org/10.1093/scipol/scv042

Caparrós, A. (1990). Notas para una reflexión sobre las relaciones entre psicología académica y psicología profesional [Notes for a reflection on the relationships between academic psychology and professional psychology]. Anuario de Psicología, 41(2), 21-30. https://raco.cat/index.php/ AnuarioPsicologia/article/view/64600

Carpintero, H. (1980). La psicología actual desde una perspectiva bibliométrica: Una introducción [Current Psychology from a Bibliometric Perspective: An Introduction]. Análisis y Modificación de Conducta, 11-12, 9-23. http://dx.doi. org/10.33776/amc.v6i11-12.1541

Carpintero, H. (1984). The impact of the Spanish Civil War on Spanish Scientific Psychology. Revista de Historia de la Psicología, 5(1-2), 91-98. https://www. revistahistoriapsicologia.es/archivo-all-issues/1984-vol-5-n\%C3\%BAm-1-2/

Carpintero, H. (2006). Historia de la Psicología en España [History of Psychology in Spain]. Madrid, España: Pirámide.

Carpintero, H. y Tortosa, F. (1996). La Psicología española a través de la Revista de Psicología General y Aplicada [Spanish Psychology through the Journal of General and Applied Psychology]. Revista de Psicología General y Aplicada, 49(3-4), 373-410. https://dialnet.unirioja.es/servlet/ articulo? codigo $=2358351$

De Filippo, D., Sanz Casado, E. y Gómez, I. (2009). Movilidad científica y género. Estudio del profesorado de una universidad española [Scientific mobility and gender. Study of the teaching staff of a Spanish university]. Revista Mexicana de Sociología, 71, 351-386. http://www.scielo.org.mx/scielo.php?script=sci_ arttext\&pid=S0188-25032009000200005

FECYT (Fundación Española para la Ciencia y la Tecnología) (2017). Indicadores del Sistema español de ciencia, tecnología e innovación [Indicators of the Spanish Science, Technology and Innovation System]. Madrid: Fecyt. http://www.idi.mine-co.gob.es/stfls/MICINN/Investigacion/FICHEROS/ Indicadores_2017.pdf

Furumoto, L. (1992). Joining separate spheres: Cristine Ladd-Franklin womanscientist (1847-1930). American Psychologist, 47, 2, 175-182. https://psycnet. apa.org/doi/10.1037/0003-066X.47.2.175

Ibarz, V. (2013). La Psicología en el Primer Congreso de la Asociación Española para el Progreso de las Ciencias (1908) [Psychology in the First Congress of the Spanish Association for the Progress of Sciences (1908)]. Revista de Historia de la Psicología, 34(3), 39-56. https://www.revistahistoriapsicologia. es/archivo-all-issues/2013-vol-34-n\%C3\%BAm-3/

García, E. (2005). Una década de transición de la Psicología en España [A decade of transition for Psychology in Spain]. Revista de Historia de la Psicología, 26(1), 101-117. https://www.revistahistoriapsicologia.es/archivo-allissues/2005-vol-26-núm-1/

García Dauder, S. (2005). Psicología y feminismo: historia olvidada de mujeres pioneras en Psicología [Psychology and feminism: Forgotten history of pioneering women in Psychology]. Madrid: Narcea.

Germain, J. (1980). Autobiografía II. Revista de Historia de la Psicología, 1(2), 139-169. https://www.revistahistoriapsicologia.es/archivo-all-issues/1980vol-1-n\%C3\%BAm-2/

González Alcaide, G. y Gómez Ferri, J. (2014). La colaboración científica: principales líneas de investigación y retos de futuro [Scientific collaboration: main research lines and future challenges]. Revista Española de Documentación Científica, 37(4): e062. doi: http://dx.doi.org/10.3989/redc.2014.4.1186

González-Alcaide, G., Castelló-Cogollos, L., Bolaños-Pizarro, M., Alonso-Arroyo, A., Valderrama-Zurián, J. C. y Aleixandre-Benavent, R. (2010). Veinte años de investigación de la psicología española en Psicothema (1989-2008) [Twenty years of Spanish psychological research in Psicothema (1989-2008)]. Psicothema, 22(1), 41-50.

González Sala, F. y Osca-Lluch, J. (2018). Desigualdad de género en órganos directivos y producción científica de las revistas iberoamericanas de psicología de mayor visibilidad internacional [Gender inequality in directive bodies and scientific production on the Ibero-American psychology journals with greater international visibility]. Revista Española de Documentación Científica, 41(3): e211. https://doi.org/10.3989/redc.2018.3.1506

González-Sala, F., Haba-Osca, J. y Osca-Lluch, J. (2021). La investigación española en psicología educativa desde una perspectiva de género (2008-2018) [Spanish research in Educational Psychology from a gender perspective (2008-2018)]. Anales de Psicología, 37(1), 44-50. https://doi.org/10.6018/ analesps.399871

González-Sala, F., Osca-Lluch, J. y Peñaranda-Ortega, M. (2021). Evolución de la colaboración científica en la Psicología española entre 1970 y 1989 [Evolution of scientific collaboration within Spanish Psychology between 1970 and 1989]. Anales de Psicología, 37(3), 589-598. https://doi.org/10.6018/ analesps. 474391

Knorr-Cetina, K. y Mulkay, M. (1983). Introducction: Emerging Principles in Social Studies of Science. In K. Knorr-Cetina \& M. Mulkay. Science Observed. Perspectives on the social Studies of Science (págs. 1-17). Sage.

López Ferrer, M. y Osca-Lluch, J. (2009). Una aproximación a la psicología en España desde el análisis de redes sociales [An approach to psychology in Spain from the analysis of social networks]. Revista de Historia de la Psicología, 30(4), 55-73. https://www.revistahistoriapsicologia.es/archivoall-issues/2009-vol-30-núm-4/

Merton, Robert K. (1977). La sociología de la ciencia: investigaciones teóricas y empíricas. Recopilación e introducción de Norman W. Storer [The sociology of science: theoretical and empirical investigations. Compilation and introduction by Norman W. Storer]. Madrid: Alianza Editorial.

Morillo, F., Bordons, M. y Gómez, I. (2003). Interdisciplinarity in science: a tentative typology of disciplines and research areas. Journal of the American Society for Information Science and Technology, 54(13), 1237-1249. http:// dx.doi.org/10.1002/asi.10326

Olivas-Ávila, J. A. y Musi-Lechuga, B. (2010). Análisis de la producción de los profesores funcionarios de Psicología en España en artículos de revistas de 
la Web of Science [Analysis of the production of psychology professors in Spain in journal articles of the Web of Science]. Psicothema, 22(4), 909-916.

Pérez-Delgado, E., Peiró, J. M. y Carpintero, H. (1981). Estudio prosopográfico de los autores eminentes para la Psicología [Prosopographic study of eminent authors for Psychology]. Revista de Historia de la Psicología, 2(3), 247-270. https://www.revistahistoriapsicologia.es/archivo-all-issues/1981-vol-2n\%C3\%BAm-3/

Rafols, I. y Meyer, M. (2007). How cross-disciplinary is bionanotechnology? Explorations in the specialty of molecular motors. Scientometrics, 70(3), 633650. http://dx.doi.org/10.1007/s11192-007-0305-3

Rafols, I. y Meyer, M. (2010). Diversity and network coherence as indicators of interdisciplinarity: case studies in bionanoscience. Scientometrics, 82(2), 263-287. http://dx.doi.org/10.1007/s11192-009-0041-y

Scarborough, E. y Furumoto, L. (1987). Untold lives. The first generation of American women psychologist. New York: Columbia University Press.
Tortosa, F. y Civera, C. (2001). Revistas y disciplina psicológica. Cien años de encuentro [Journals and psychological discipline. One hundred years of encounter]. Papeles del Psicólogo, 79. http://www.papelesdelpsicologo.es/ resumen?pii=858

Tortosa-Pérez, M., Santolaya Prego de Oliver, J., Santolaya, F. y Tortosa, F. (2021). Psicología y profesión en España. Los primeros años del Colegio Oficial de Psicólogos [Psychology and profession in Spain. The first years of the Official Association of Psychologists]. Papeles del Psicólogo, 42(2), 105. https://doi. org/10.23923/pap.psicol.2961

Vera, J. A. (2005). 1979 Un año para recordar en la Historia de la Psicología española [1979 A year to remember in the History of Spanish Psychology]. Revista de Historia de la Psicología, 26(4), 213-242. https://www. revistahistoriapsicologia.es/archivo-all-issues/2005-vol-26-núm-4/

Wilson, D. A. H. (2003). British female academics and comparative psychology: Attempts to establish a research niche in the early 20th century. History of Psychology, 6(1), 89-109. https://doi.org/10.1037/1093-4510.6.1.89 